\title{
On certain shallow water models, scaling invariance and strict self-adjointness
}

\author{
Priscila Leal da Silva, Igor Leite Freire, \\ Centro de Matemática, Computação e Cognição, CMCC, UFABC, \\ 09210-580, Santo André, SP \\ E-mail: priscila.silva@ufabc.edu.br, igor.freire@ufabc.edu.br.
}

\begin{abstract}
Resumo: In this work we establish conditions for a class of third order partial differential equations to be strictly self-adjoint and scale invariant. The obtained family of equations includes the Benjamin-Bona-Mahony, Camassa-Holm and Novikov equations. Using the strict selfadjointness and Ibragimov's conservation theorem, we establish some local conservation laws for some of the mentioned equations.
\end{abstract}

Palavras-chave: Strict self-adjointness, Ibragimov's conservation theorem, conservation laws.

\section{Historical survey}

During the last century, a sequence of papers, starting with [27], showed and enlightened many properties of the well known Korteweg - de Vries equation

$$
u_{t}=u_{x x x}+u u_{x} .
$$

Later, in [1], a new equation called Benjamin-Bona-Mahoney (BBM), given by

$$
u_{t}=u_{t x x}+u u_{x}
$$

was derived as an "alternative" for the KdV.

Although equation (2) was derived using the same formal justification for obtaining (1), the differences between both equations are greater than the fact that (1) is an evolution equation whereas (2) is not. In [1] the authors found three conserved quantities on the solutions of (2). Later in [30], those conservation laws obtained were proved to be the only three admitted by (2). This fact shows a dramatic difference between BBM and KdV since the last one admits an infinite number of conserved quantities [28].

More recently, Camassa and Holm [5] using Hamiltonian methods derived the famous CamassaHolm $(\mathrm{CH})$ equation

$$
u_{t}-u_{t x x}+3 u u_{x}=2 u_{x} u_{x x}+u u_{x x x} .
$$

The last equation possesses remarkable properties such as peakon solutions and a bi-hamiltoninan structure, see [5], which implies in the existence of an infinite number of conserved quantities, just like the $\mathrm{KdV}$ equation [15, 28, 26].

Since then, a considerable number of papers have been dedicated to derive third order nonevolutionary dispersive equations having similar properties as those known to $\mathrm{KdV}$ and $\mathrm{CH}$ equation. To cite a few number of examples, it was derived in [8] an integrable equation having peakon solutions with first order nonlinearities, while in [9] another integrable equation, combining linear dispersion such as the $\mathrm{KdV}$ equation and a nonlinear dispersion like the $\mathrm{CH}$ 
equation, was discovered. In [8] an integrable equation with peakon solutions was considered and, more recently, Novikov [29] has discovered the equation

$$
u_{t}-u_{t x x}+4 u^{2} u_{x}=3 u u_{x} u_{x x}+u^{2} u_{x x x}
$$

which not only admits peakon solutions and cubic nonlinearities, but it is also integrable [17].

In [28] it was shown that the KdV equation possesses infinitely many conservation laws. This was the start point of a considerable number of papers dealing with the properties of a certain equation and the existence of an infinite number of conserved quantities.

Noether theorem showed a deeper and closer relation between symmetries and conservation laws for the Euler-Lagrange equations. She showed that for each conservation law of a differential equation, or system, there is a symmetry property related to it. However, Noether theorem requires that the equation is an Euler-Lagrange equation, and although the KdV equation is not an Euler-Lagrange equation, it can be transformed in one using the differential substitution $u=v_{x}$. Then, from it, one arrives at the equation $v_{t x}=v_{x x x x}+v_{x} v_{x x}$, which is an EulerLagrange equation.

The first paper relating symmetries (not necessarily Lie point symmetries) of the KdV equation and an infinite number of local conservation laws for it was [21], in which Ibragimov showed how to construct local conservation laws using symmetries other than the Lie point symmetries.

In order to construct the conserved vectors, Ibragimov first established a non-local conserved vector. Then he showed that the $\mathrm{KdV}$ equation is strictly self-adjoint $[20,21,25]$ and, consequently, the non-local conserved quantities can be transformed in locals one. A considerable number of integrable equations has a common property: strict self-adjointness.

Ibragimov in [21] showed that KdV is strictly self-adjoint. In [24] it was shown that the $\mathrm{CH}$ equation has also the same property, as well as in [4] it was proved that the Novikov equation is strictly self-adjoint. In particular, with respect to (3) and (4), the obtained results in [6], [24] and [4] shows some common facts:

1. both equations are strictly self-adjoint;

2. both equations admit the scaling symmetry $(x, t, u) \mapsto\left(x, \lambda^{-b} t, \lambda u\right)$, for a certain value of $b$, whose corresponding generator is

$$
X_{b}=u \frac{\partial}{\partial u}-b t \frac{\partial}{\partial t}
$$

Since Ibragimov's concepts on self-adjointness have been introduced, a considerable number of papers has been dealing with the problem of finding classes of differential equations with some self-adjoint property, see, for instance, [10, 11, 12, 13, 14, 16, 32].

Therefore, motivated by those recent results and provoked by the classification carried out in [29], in which certain generalizations of the $\mathrm{CH}$ equation possessing infinite hierarchies of higher symmetries were considered, we tried to determine which conditions are necessary and sufficient for the equation

$$
F=u_{t}+\epsilon u_{t x x}+f(u) u_{x}+g(u) u_{x} u_{x x}+h(u) u_{x x x}=0
$$

to be strictly self-adjoint. After that we restrict ourselves to find a subclass of (6) that admits a certain scaling symmetry. Then we can find local conservation laws using the conservation theorem Ibragimov proposed in recent years. Next sections will talk about theory and original results obtained. All results presented here can be found more detailed in [7].

\section{Strict self-adjointness and invariance}

According to Ibragimov [21, 22, 23, 25], a differential equation

$$
F\left(x, u, u_{(1)}, \ldots, u_{(n)}\right)=0
$$


is said to be strictly self-adjoint if, and only if,

$$
\left.F^{*}\right|_{v=u}=\lambda F,
$$

for some differential function $\lambda$, where $x \in \mathbb{R}^{n}, u_{(i)}$ denotes the derivatives of $u$ of order $i$, and $F^{*}$ is the adjoint equation:

$$
F^{*}:=\frac{\delta}{\delta u}(v F)
$$

where the Euler Lagrange operator $\frac{\delta}{\delta u}$ is given by the formal sum

$$
\frac{\delta}{\delta u}=\frac{\partial}{\partial u}+\sum_{j=1}^{\infty}(-1)^{j} D_{i_{1}} \ldots D_{i_{j}} \frac{\partial}{\partial u_{i_{1} \ldots i_{j}}} .
$$

Theorem 2.1. Equation (6) is strictly self-adjoint if and only if

$$
g(u)=\frac{(u h)^{\prime}}{u}+\frac{c}{u}
$$

where $c$ is an arbitrary constant.

To prove the theorem we have to first find the adjoint equation $F^{*}=0$, which is given by

$$
\begin{gathered}
F^{*}=v\left[g^{\prime}(u) u_{x} u_{x x}+f^{\prime}(u) u_{x}+h^{\prime}(u) u_{x x x}\right]-D_{t}(v)-D_{x}\left[v\left(f(u)+g(u) u_{x x}\right)\right]+ \\
+D_{x}^{2}\left[v g(u) u_{x}\right]-D_{x}^{2} D_{t}(\epsilon v)-D_{x}^{3}[v h(u)] .
\end{gathered}
$$

Using condition (8), it is found that $\lambda=-1$ and the following constraints, arising from the coefficients of $u_{x}^{3}$ and $u_{x} u_{x x}$, respectively:

$$
\begin{aligned}
& (u g)^{\prime \prime}-(u h)^{\prime \prime \prime}=0 \\
& (u g)^{\prime}-(u h)^{\prime \prime}=0 .
\end{aligned}
$$

Clearly the last condition implies the first one and integrating it once, we obtain the desired result.

We can now restrict ourselves to find the subfamily of strictly self-adjoint equations admitting the scaling symmetry $(x, t, u) \mapsto\left(x, \lambda^{-b} t, \lambda u\right)$.

We recall that a differential equation (7) admits a symmetry $(x, u) \mapsto(\hat{x}, \hat{u}), \quad x \in \mathbb{R}^{n}$ if its corresponding infinitesimal generator

$$
X=\xi(x, y) \frac{\partial}{\partial x}+\eta(x, y) \frac{\partial}{\partial y}
$$

satisfies the condition

$$
X^{(n)} F=\sigma F,
$$

where $X^{(n)}$ is the $n$-th prolongation of $X$, see $[2,3,18,19,31]$, and $\sigma$ is a suitable differential function.

Using Theorem 2.1 and the invariance condition $X^{(3)} F=\sigma F$, we obtain explicit conditions for funtions $f(u), g(u)$ and $h(u)$ by having the following four-parameter family of scale invariant strictly self-adjoint equations

$$
u_{t}+\epsilon u_{t x x}+\gamma u^{b} u_{x}=(1+b) \beta u^{b-1} u_{x} u_{x x}+\beta u^{b} u_{x x x}
$$


which includes equations (2), (3) and (4). Moreover, taking $b=1, \epsilon=-\beta=\alpha^{2}$ and $\gamma=3$, we arrive, up to a translation $u \mapsto u+u_{0} / \alpha^{2}$, at the Dullin-Gotwald-Holm equation

$$
u_{t}-\alpha^{2} u_{t x x}+3 u u_{x}=\alpha^{2}\left(u u_{x x x}+2 u_{x} u_{x x}\right)+u_{0} u_{x x x}
$$

which is also integrable, see [9]. The term $u_{0}$ corresponds to the coefficient of the linear dispersion of the equation and when $u_{0} \rightarrow 0$ and $\alpha=1$, such equation turns back to the $\mathrm{CH}$ equation. However, if $u_{0} \neq 0,(11)$ does not admit the generator (5). For instance, when $u_{0}=1$ and $\epsilon \rightarrow 0$, one easily obtains the $\mathrm{KdV}$ equation, and it is not scale invariant. We also observe that at the limit of the dispersionless $u_{0}, \alpha \rightarrow 0$, equation (11) is reduced to the Riemann equation $u_{t}+3 u u_{x}=0$. More generally, when the dispersion effects are neglected in (10), that is, $\epsilon, \beta \rightarrow 0$, one obtains a family of Riemann equations given by

$$
u_{t}+\gamma u^{b} u_{x}=0
$$

\section{Conservation laws}

Ibragimov in $[20,21,22,23,25]$ established connections between a system of differential equations, formed by a differential equation $F=0$ and its adjoint $F^{*}=0$, and the theorem proved by Noether. He showed that a symmetry admitted by an equation is inherited by its adjoint equation. Moreover, he proved that this symmetry is a variational symmetry for the system, with the Lagrangean $\mathcal{L}=v F$, and then one can find non-local conservation laws using Noether theorem.

For this particular case, Noether theorem states thay

$$
\begin{aligned}
C^{0}= & \tau \mathcal{L}+W\left[\frac{\partial \mathcal{L}}{\partial u_{t}}+D_{x}^{2}\left(\frac{\partial \mathcal{L}}{\partial u_{t x x}}\right)\right]-D_{x}(W) D_{x}\left(\frac{\partial \mathcal{L}}{\partial u_{t x x}}\right)+D_{x}^{2}(W) \frac{\partial \mathcal{L}}{\partial u_{t x x}}, \\
C^{1}= & \xi \mathcal{L}+W\left[\frac{\partial \mathcal{L}}{\partial u_{x}}-D_{x}\left(\frac{\partial \mathcal{L}}{\partial u_{x x}}\right)+D_{x}^{2}\left(\frac{\partial \mathcal{L}}{\partial u_{x x x}}\right)+D_{x} D_{t}\left(\frac{\partial \mathcal{L}}{\partial u_{x x t}}\right)+D_{t} D_{x}\left(\frac{\partial \mathcal{L}}{\partial u_{x t x}}\right)\right] \\
& D_{x}(W) D_{x}\left[\frac{\partial \mathcal{L}}{\partial u_{x x}}-D_{x}\left(\frac{\partial \mathcal{L}}{\partial u_{x x x}}\right)-D_{t}\left(\frac{\partial \mathcal{L}}{\partial u_{x x t}}\right)\right]-D_{t}(W) D_{x}\left(\frac{\partial \mathcal{L}}{\partial u_{x t x}}\right)+D_{x}^{2}(W) \frac{\partial \mathcal{L}}{\partial u_{x x x}} \\
& +D_{t} D_{x}(W) \frac{\partial \mathcal{L}}{\partial u_{x t x}}+D_{x} D_{t}(W) \frac{\partial \mathcal{L}}{\partial u_{x x t}},
\end{aligned}
$$

provides a conserved current for our equation, where $W=\eta-\xi u_{x}-\tau u_{t}$ and the formal Lagrangian $\mathcal{L}$ is given by

$$
\mathcal{L}=v\left[u_{t}+\epsilon \frac{u_{t x x}+u_{x t x}+u_{x x t}}{3}+f(u) u_{x}+g(u) u_{x} u_{x x}+h(u) u_{x x x}\right] .
$$

With the concept of strict self-adjointness, it is possible to remove the non-local variable $v$ that arises from the definition of the Lagrangean $\mathcal{L}$ by setting $v=u$, see [23, 25]. That being, the adjoint equation is then equivalent to the original equation and Noether theorem provides local conservation laws for the equation initially considered. There are generalizations of this strict self-adjoitness concept, but here are of no interest right now to our studies presented here.

For the family (10) of strictly self-adjoint equations, the scale symmetry generator (5) yields the conserved current of components

$$
C^{0}=u^{2}-\epsilon u_{x}^{2}, \quad C^{1}=\frac{2}{2+b} \gamma u^{b+2}-2 \beta u^{b+1} u_{x x}+2 \epsilon u u_{t x} .
$$

The conserved density $C^{0}$ of (15) is valid for all values of $b$, while the conserved flux $C^{1}$ corresponds to the cases when $b \neq-2,0$. 
Also, the conservation law of components given by (15) includes quantities known in the literature, coming from generator (5), for equations (2), (3) and (4). The conserved vector (15) also provides a known conservation law for the class of Riemann equations (12), but only if $b \neq-2$.

In [7], we also found the conservation currents for cases $b=0$ and $b=-2$. The component $C^{0}$ remains unchanged, while the component $C^{1}$, for $b=0$ and $b=-2$, is given respectively by

$$
\begin{gathered}
C_{0}^{1}=\gamma u^{2}-2(\beta-c) u u_{x x}+2 \epsilon u u_{t x}-c u_{x}^{2}, \\
C_{-2}^{1}=-2 \beta \frac{u_{x x}}{u}+2 \gamma \ln u+2 \epsilon u u_{x t} .
\end{gathered}
$$

That way, for all values of $b$ one can obtain the conservation law arising from a scale symmetry for equation (10), a class of equations that contains many of the most famous shallow water models studied.

Acknowledgements: The authors would like to thank FAPESP for financial support, project numbers 2012/22725-4 and 2011/19089-6, and CNPq for finanancial support, project number $308941 / 2013-6$.

\section{Referências}

[1] T. B. Benjamin, J. L. Bona and J. J. Mahony, Model equations for long waves in nonlinear dispersive systems, Philos. Trans. Roy. Soc. London, 272 (1972) 47-78.

[2] G. W. Bluman and S. Anco, "Symmetry and Integration Methods for Differential Equations", Springer, New York, 2002.

[3] G. W. Bluman and S. Kumei, "Symmetries and Differential Equations", Applied Mathematical Sciences 81, Springer, New York, 1989.

[4] Y. Bozhkov, I. L. Freire and N. H. Ibragimov, Group analysis of the Novikov equation, Comp. Appl. Math., DOI 10.1007/s40314-013-0055-1, 2013.

[5] T. Camassa and D. D. Holm, An integrable shallow water equation with peaked solitons, Phys. Rev. Lett., 71 (1993) 1661-1664.

[6] P. A. Clarkson, E. L. Mansfield and T. J. Priestley, Symmetries of a class of nonlinear third-order partial differential equations, Math. Comput. Modelling, 25 (1997) 195-212.

[7] P. L. da Silva and I. L. Freire, Strict self-adjointness and shallow water models, arXiv:1312.3992v1, 2013.

[8] A. Degasperis, D. D. Holm and A. N. W. Hone, A new integrable equation with peakon solutions, Theor. Math. Phys., 133 (2002) 1463-1474.

[9] R. R. Dullin, G. A. Gottwald and D. D. Holm, An integrable shallow water equation with linear and nonlinear dispersion, Phys. Rev. Lett., 87 (2001) 194501, 4pp.

[10] I. L. Freire, Conservation laws for self-adjoint first order evolution equations, J. Nonlin. Math. Phys., 18 (2011) 279-290.

[11] I. L. Freire, Self-adjoint sub-classes of third and fourth-order evolution equations, Appl. Math. Comp., 217 (2011) 9467-9473.

[12] I. L. Freire and J. C. S. Sampaio, Nonlinear self-adjointness of a generalized fifth-order KdV equation, J. Phys. A: Math. Theor., 45 (2012) 032001. 
[13] I. L. Freire, New conservation laws for inviscid Burgers equation, Comp. Appl. Math., 31 (2012) 559-567.

[14] I. L. Freire, New classes of nonlinearly self-adjoint evolution equations of third- and fifthorder, Commun. Nonlinear Sci. Numer. Simul., 18 (2013) 493-499.

[15] C. S. Gardner, Kortewerg-de Vries equation and generalizations IV. The Korteweg-de Vries equation as a Hamiltonian system, J. Math. Phys., 12 (1971) 1548-1551.

[16] M. L. Gandarias, Weak self-adjoint differential equations, J. Phys. A, 44 (2011) 262001, 6 pp.

[17] A. N. W. Hone and J. P, Wang, Integrable peak on equations with cubic nonlinearities, J. Phys. A: Math. Theor., 41 (2008) 372002, 10 pp.

[18] N. H. Ibragimov, "Transformation groups applied to mathematical physics", Translated from the Russian Mathematics and its Applications (Soviet Series), D. Reidel Publishing Co., Dordrecht, 1985.

[19] N. H. Ibragimov, "Elementary Lie Group Analysis and Ordinary Differential Equations", John Wiley and Sons, Chirchester, 1999.

[20] N. H. Ibragimov, Integrating factors, adjoint equations and Lagrangians, J. Math. Anal. Appl., 318 (2006) 742-757.

[21] N. H. Ibragimov, A new conservation theorem, J. Math. Anal. Appl., 333 (2007) 311-328.

[22] N. H. Ibragimov, Quasi-self-adjoint differential equations, Archives of ALGA, 4 (2007) 5560.

[23] N. H. Ibragimov, Nonlinear self-adjointness and conservation laws, J. Phys. A: Math. Theor., 44 (2011) 432002, 8 pp.

[24] N.H. Ibragimov, R.S. Khamitova, A. Valenti, Self-adjointness of a generalized CamassaHolm equation, Appl. Math. Comp., 218 (2011) 2579-2583.

[25] N. H. Ibragimov, Nonlinear self-adjointness in constructing conservation laws, Archives of ALGA, 7/8 (2011) 1-90.

[26] M. D. Kruskal, R. M. Miura, C. S. Gardner and N. J. Zabusky, Korteweg-de Vries equation and generalizations. V. Uniqueness and nonexistence of polynomial conservation laws, $J$. Math. Phys., 11 (1970) 952-960.

[27] R. M. Miura, Korteweg-de Vries equation and generalizations. I. A remarkable explicit nonlinear transformation, J. Math. Phys., 9 (1968) 1202-1204.

[28] R. M. Miura, C. S. Gardner and M. D. Kruskal, Korteweg-de Vries equation and generalizations. II. Existence of conservation laws and constants of motion, J. Math. Phys., 9 (1968) 1204-1209.

[29] V. S. Novikov, Generalizations of the Camassa-Holm equation, J. Phys. A: Math. Theor, 42 (2009) 342002, 14pp.

[30] P. J. Olver, Euler operators and conservation laws of the BBM equation, Math. Proc. Cambridge Phils. Soc., vol. 85 (1979) 143-160.

[31] P. J. Olver, "Applications of Lie groups to differential equations", Springer, New York, 1986.

[32] M. Torrisi and R. Tracinà, Quasi self-adjointness of a class of third order nonlinear dispersive equations, Nonlin. Anal. RWA, 14 (2013). 